\title{
The natural history of autoimmune Addison's disease from the detection of autoantibodies to development of the disease: a long-term follow-up study on 143 patients
}

\section{Lara Naletto", Anna Chiara Frigo², Filippo Ceccato', Chiara Sabbadin', Riccardo Scarpa1, Fabio Presotto³, Miriam Dalla Costa', Diego Faggian4, Mario Plebani ${ }^{4}$, Simona Censi $^{1}$, Jacopo Manso', Jadwiga Furmaniak ${ }^{5}$, Shu Chen ${ }^{5}$, Bernard Rees Smith5, Stefano Masiero', Francesca Pigliaru', Marco Boscaro ${ }^{1}$, Carla Scaroni ${ }^{1}$ and Corrado Betterle ${ }^{1}$}

${ }^{1}$ Endocrine Unit, Department of Medicine (DIMED), ${ }^{2}$ Unit of Biostatistic, Epidemiology and Public Health, Department of Cardiac, Thoracic and Vascular Sciences, University of Padova, Padova, Italy, ${ }^{3}$ Unit of Internal Medicine, Ospedale dell'Angelo, Mestre-Venezia, Italy, ${ }^{4}$ Unit of Laboratory Medicine, Department of Medicine (DIMED), University of Padua, Padua, Italy, ${ }^{5}$ FIRS Laboratories, RSR Ltd, Parc Ty Glas, Llanishen, Cardiff, UK, and ${ }^{6}$ Endocrine Unit, Department of Medical Sciences, University of Cagliari, Cagliari, Italy

Correspondence should be addressed to $\mathrm{C}$ Betterle

Email

corrado.betterle@unipd.it

\begin{abstract}
Background: Adrenal cortex autoantibodies (ACAs) and/or 21-hydroxylase (21OHAb) are markers of autoimmune Addison's disease (AAD) and progression to overt AAD. The reported cumulative risk of developing AAD varies from 0 to $90 \%$ in different studies.

Aim: To assess the predictive value of different parameters in the progression toward AAD in patients with ACA and/or 21OHAb-positive patients with autoimmune polyendocrine syndromes (APS).

Materials and methods: Twenty-nine patients with APS-1 and 114 patients with APS-2 or APS-4 were followed up for a median of 10 years (range 6 months to 33 years) and were assessed using ACTH test. The risk of AAD was estimated according to age, gender, stage of adrenal dysfunction, associated diseases and antibody titer. Univariate and multivariate Cox proportional hazard models were used for statistical analysis.

Results: The cumulative risk (CR) of developing AAD was higher in APS-1 patients (94.2\%) than in patients with APS-2/APS-4 (38.7\%). The CR was high in both male and female APS-1 patients, while in patients with APS-2/APS-4 it was high only in males. Stage 1 (increased plasma renin) for patients with APS-1 and Stage 2 (no response of cortisol to ACTH test) for patients with APS-2/APS-4 were established as the points of no return in the progression to AAD. Adjusted hazard ratio analyses by multivariate Cox model for AAD showed that gender, diseases and adrenal function were independent risk factors for developing clinical AAD. The risk of developing clinical AAD appears to subside after 19 years of follow-up.

Conclusions: A model for estimating the probability to survive free of AAD has been developed and should be a useful tool in designing appropriate follow-up intervals and future therapeutic strategies.
\end{abstract}




\section{Introduction}

Primary adrenal failure, or Addison's disease (AD), is characterized by deficiency of the adrenocortical hormones cortisol, aldosterone and androgens, with high plasma levels of ACTH and plasma renin activity (PRA) $(1,2)$. It is a rare disorder, with a prevalence estimated from 110 to 144 per million and an incidence of $4.4-5.6$ new cases per million per year (3). The highest prevalence has recently been reported in Iceland with 221 cases per million (4). In developed countries, autoimmune AD (AAD) is the most common cause of primary adrenal failure. It is responsible for about $80 \%$ of $\mathrm{AD}$ in the adult population and can occur as an isolated disease or, more frequently, associated with other autoimmune diseases classed in three types of autoimmune polyendocrine syndromes (APS). Type 1 (APS-1) is a very rare disease caused by the mutation of AIRE gene manifesting with at least two of the three main component diseases: chronic candidiasis (CC), chronic hypoparathyroidism $(\mathrm{CH})$ and AAD. Type 2 (APS-2) is an HLA-linked syndrome less rare than APS- 1 and is characterized by AAD associated with autoimmune thyroid diseases (AITD) and/or type 1 diabetes mellitus (DM-1). In type 4 (APS-4), AAD presents in association with other autoimmune diseases (OAD) not included in APS-1 or APS-2 (2, 5). There are differences of opinion regarding this detailed classification and some authors prefer to classify AAD as isolated or associated in the context of two main syndromes (APS-1 or APS-2) (6, 7).

It should be noted that the presentation of APS- 1 is not always limited to the three main component diseases. The clinical presentation can be more heterogeneous and include enamel hypoplasia, enteropathy with chronic diarrhea or constipation, premature ovarian insufficiency, periodic fever with rash, non-infectious keratitis or autoimmune hepatitis $(7,8,9)$.

Adrenal cortex autoantibodies (ACA), measured using the indirect immunofluorescence test, were first described in 1962 in patients with so-called idiopathic $\mathrm{AD}$ (10), and for many years, this technique was used to identify patients with clinical AAD or patients and individuals with potential AAD $(2,11)$. In 1992, steroid 21-hydroxylase $(21 \mathrm{OH})$ was identified as the main target autoantigen recognized by ACA $(12,13)$ and, in addition to ACA, autoantibodies to $21 \mathrm{OH}$ (21OHAb) have become the gold standard for the diagnosis, monitoring and prediction of $\operatorname{AAD}(2,5,11)$.

ACA and/or 21OHAbs were reported in $0-15 \%$ of 23480 patients with non-adrenal autoimmune diseases, as previously reviewed $(2,11)$. Specifically, they were found in $0-3 \%$ of patients with $\operatorname{AITD}(14,15,16)$ or in $0.4-2.3 \%$ of those with DM-1 $(14,15,16,17,18,19$, 20). ACA and/or 21 OHAbs were found in $48 \%$ of young APS-1 patients (14) and in 9\% of females with premature ovarian insufficiency (POI) $(2,5,11)$. Also, they have been reported in 5\% (range $0-8 \%$ ) of 138 first-degree relatives of AAD patients (11) and in 4.1\% (range $0-5.3 \%$ ) of 1273 hospitalized patients (11), as well as in $0.6 \%$ (range $0.06-$ $1.6 \%$ ) of 6488 normal controls (11). Recently, using a new and more sensitive ELISA, the presence of 210 HAbs has been shown in $2 \%$ of 49 adult patients with DM-1. Moreover, $4.2 \%$ of 120 children with AITD and $2.5 \%$ of 119 children with DM-1 were found to be positive for 210 HAbs. In addition, 210 HAbs were found in $0.6 \%$ of 928 healthy adult blood donors, but in none of the 108 adult patients with OAD (21).

The diagnostic and predictive value of ACA/21OHAbs in the natural history of AAD has been recognized in different studies, and the reported rate of progression to overt disease varied from 0 to $90 \%$, depending on the number of patients, their age, gender, adrenal function, HLA pattern and type of non-adrenal disease present at the start of follow-up $(2,11)$. In particular, the risk of developing AAD reported in different studies was higher (from $74 \%$ to $90 \%$ ) in patients with APS-1 $(14,22,23)$ than in $0-44 \%$ of patients with APS-2, or APS-4 $(11,15$, $17,18,24,25,26)$ and virtually absent in hospitalized patients $(11,27)$. In a previous study comprising 100 ACA/21OHAb-positive patients followed for a median period of 4.8 years (range 3 months to 21 years), we found that $31 \%$ of these patients developed $\mathrm{AAD}$ and that the development of AAD was associated with four independent risk factors, i.e., male gender, impaired adrenal function at entry into the study, high titer of ACA/21OHAbs and pre-existing autoimmune diseases of which $\mathrm{CH}$ and/or CC (APS-1) were associated with a very high risk (20). Based on these results, we produced an algorithm for estimating low, intermediate or high risk of developing $\mathrm{AAD}$ and proposed optimum interval periods for assessing the adrenal function in ACA/21OHAbpositive patients. The study also suggested that the point of no return toward clinical AAD was at Stage 1 of subclinical adrenal insufficiency and is characterized by increased PRA or plasma renin concentration with normal or low levels of aldosterone. Furthermore, the longest time of progression to AAD was 11 years from the start of follow-up $(14,15,26,28)$.

The aim of the present study was to review the previous data using a greater number of asymptomatic 
patients with ACA/21OHAbs followed for a longer period and to re-evaluate the risk of progression to clinically overt AAD. Furthermore, patients with APS-1 were analyzed separately from those with OADs (i.e. APS-2 or APS-4).

\section{Subjects and methods}

\section{Patients and controls}

We enrolled 143 ACA/21OHAb-positive patients who did not present with AAD at the time of entry into the study and followed them for a median of 10 years (range 6 months to 33 years). Among these patients, 28 initially presented with APS-1 and 114 presented with OADs (98 with APS-2 and 16 with APS-4). As controls, we followed 106 ACA and/or 21OHAb-negative patients matched for age, gender and autoimmune diseases, and they were tested for basal ACTH, cortisol and PRA and followed for a median period of 6 years (range 1-30 years). Ten patients in the control group were affected by APS- 1 and one of them seroconverted for ACA during follow-up and was included in the group of ACA/21OHAb-positive patients with APS-1, increasing the final number of patients to 29. The main features of the followed ACA/21OHAbpositive patients and ACA/21OHAb-negative controls are summarized in Table 1.

During follow-up, patients with DM-1 received insulin therapy, patients with $\mathrm{CH}$ calcium and calcitriol and patients with POI hormone replacement therapy. Patients with active Graves' disease were treated with methimazole or propylthiouracil and those with hypothyroidism received levothyroxine. Seven patients received immunosuppressive or immunomodulating therapies: one for rheumatoid arthritis, two for chronic urticaria, one for psoriasis, one for Graves' ophthalmopathy, one for sero-negative arthritis and one for kidney and pancreas transplantation.

Twenty-eight ACA/21OHAb-positive and 10 ACA/21OHAb-negative patients with APS-1 were tested for AIRE gene mutations using the method described previously (29).

The Ethics Committee of Padova University-Hospital approved the study protocol, and all patients gave written informed consent after full explanation of the purpose and nature of all procedures used.

\section{Tests of adrenal function}

Adrenal cortex function was evaluated by a high dose

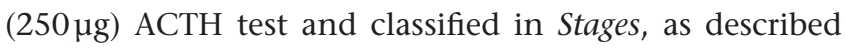

previously $(24,26)$. Cortisol levels were measured at $60 \mathrm{~min}$ post ACTH intravenous injection with a cut-off for normal values above $500 \mathrm{nmol} / \mathrm{L}$. Cortisol was measured using radioimmunoassay method as described previously (24, 26) and since the year 2006 using a solid phase competitive chemiluminescent enzyme immunoassay (Immunolite 2000 Cortisol, Siemens Healthcare Diagnostics Products Ltd., Gwynedd, UK). In summary, Stage 0 was defined by

Table 1 Clinical features of the 143 ACA/21OHAb-positive patients and $106 \mathrm{ACA} / 210 \mathrm{HAb}$-negative controls enrolled in the follow-up study.

\begin{tabular}{|c|c|}
\hline Clinical features & Values \\
\hline ACA/21OHAb-positive patients, total $n$ & 143 \\
\hline Mean age and range at entry & 31 years (4-75 years) \\
\hline Young patients ( $\leq 18$ years), $n$ & 33 \\
\hline With APS-1 & 20 \\
\hline Without APS-1 & 13 \\
\hline Adult patients ( $>18$ years), $n$ & 110 \\
\hline With APS-1 & 9 \\
\hline Without APS-1 & 101 \\
\hline Median period of follow-up (range) & $\begin{array}{c}10 \text { years ( } 6 \text { months to } \\
33 \text { years) }\end{array}$ \\
\hline Quartiles & $\begin{aligned}(\mathrm{Q} 1 & =5 \text { years } \\
\text { Q3 } & =17 \text { years })\end{aligned}$ \\
\hline Females/males (F/M) & $120 / 23$ \\
\hline $\begin{array}{l}\text { Patients with APS-1 (28 initially } \\
\text { ACA-positive and } 1 \text { seroconverted } \\
\text { during follow-up), } n\end{array}$ & 29 \\
\hline Mean age and range at entry & 15 years (4-51 years) \\
\hline F/M & $19 / 10$ \\
\hline Patients with APS- 2 or APS- 4 , total $n$ & 114 \\
\hline With APS-2, $n$ & 98 \\
\hline AITD alone & 78 \\
\hline DM-1 alone & 13 \\
\hline AITD and DM-1 & 7 \\
\hline Mean age and range at entry & 35 years (8-75 years) \\
\hline F/M & $90 / 8$ \\
\hline $\begin{array}{l}\text { With APS-4 (e.g. POI, vitiligo, } \\
\text { autoimmune hepatitis, alopecia, } \\
\text { autoimmune gastritis), } n\end{array}$ & 16 \\
\hline Mean age and range at entry & 32 years (12-62 years) \\
\hline F/M & $11 / 5$ \\
\hline ACA/21OHAb-negative patients, total $n$ & 106 \\
\hline F/M & $9 / 1$ \\
\hline Mean & 32 years (6-70 years) \\
\hline Young patients ( $\leq 18$ years), $n$ & 25 \\
\hline Adult patients ( $>18$ years), $n$ & 81 \\
\hline $\mathrm{M} / \mathrm{F}$ & $3 / 1$ \\
\hline Median period of follow-up & $\begin{array}{r}6 \text { years (range: } \\
1-30 \text { years) }\end{array}$ \\
\hline Pati & 10 \\
\hline Patients with AITD and/or DM-1, $n$ & 84 \\
\hline Patients with OAD, $n$ & 12 \\
\hline
\end{tabular}

ACA, adrenal cortex autoantibodies; AITD, autoimmune thyroid diseases; APS, autoimmune polyendocrine syndromes; DM-1, type 1 diabetes mellitus; OAD, other autoimmune diseases; POI, premature ovarian insufficiency. 
the presence of ACA and/or 21OHAbs and normal adrenal cortex function tests (baseline ACTH, PRA, aldosterone and cortisol levels and peak cortisol value after ACTH stimulation test). Stage 1 was defined by increased PRA (above the normal reference values) with normal/low levels of aldosterone and normal values of the other hormones. Stage 2 was defined by increased PRA with normal/low levels of aldosterone, normal basal levels of ACTH and cortisol, but impaired response of cortisol (below $500 \mathrm{nmol} / \mathrm{L}$ ) to intravenous ACTH. Stage 3 was defined by increased PRA with low levels of aldosterone (below the normal reference values), increased basal ACTH values (above the normal reference values) with normal or low basal cortisol levels (below the normal reference values) without response to intravenous ACTH. Stage 4 was characterized by high levels of PRA (i.e., more than two times the upper reference values) with low levels of aldosterone, very high levels of ACTH (more than two times the upper reference values), low basal cortisol levels and clinical signs and symptoms of $\operatorname{AAD}(24,26)$. The stages of adrenal impairment disclosed in the natural history of AAD by ACTH test are summarized in Table 2 . None of the patients who were assessed for adrenal function using the ACTH test were on any medication interfering with PRA (i.e., with angiotensin-converting enzymes inhibitors) and/or other measurements. In patients with Stage 1, elevated PRA was reassessed periodically (approximately every year) to confirm the diagnosis.

Patients at Stage 3 (Table 2) did not in general reveal classical symptoms or signs of $\mathrm{AD}$, although some presented with non-specific symptoms. For example, skin hyperpigmentation is known to appear after many months of increased ACTH $(26,28)$. However, considering the high risk of developing clinical AAD, particularly in case of stressful events, all patients at Stage 3 were advised to start glucocorticoid substitutive therapy $(25 \mathrm{mg}$ of cortisone acetate or $20 \mathrm{mg}$ of hydrocortisone subdivided in two or three daily doses) to avoid adrenal crisis. All patients at Stage 4 were receiving glucocorticoid and mineralocorticoid as standard replacement therapy.

\section{Adrenal autoantibodies}

ACAs were detected by the classical indirect immunofluorescence technique on normal human adrenal tissue using goat antihuman IgG conjugated to fluorescein isothiocyanate, as previously reported $(14,15$, 26). Sera showing staining of adrenal tissue at 1:2 dilution were considered as positive. Titers of ACA were defined by doubling dilution up to the end point. 21 OHAbs were measured using an immunoprecipitation assay based on ${ }^{125}$ I-labeled recombinant human $210 H$ (RSR Ltd., Cardiff, UK) as described before (26). In this assay, 21OHAb levels above $1 \mathrm{U} / \mathrm{mL}$ were considered positive (26). In the case of some patients, 210 HAbs could not be measured at entry because the assay had not yet become available. However, the serum samples obtained at enrollment were stored and tested for 21OHAbs later when possible. At enrollment, the patients were divided according to ACA titers and 21OHAb levels into two groups: the first group included patients with low-medium autoantibody levels (1:2-1:32 for ACA, and 1-100 U/mL for 21OHAbs), whereas the second group included patients with high autoantibody levels (titers $\geq 1: 64$ for ACA and/or $>100 \mathrm{U} /$ $\mathrm{mL}$ for $21 \mathrm{OHAbs}$ ). The patients with high titer ACA and low-medium $21 \mathrm{OHAb}$ levels or vice versa were assigned to the high-level group.

\section{Statistical analysis}

Data were collected on an Excel spreadsheet and analyzed with SAS 9.4 (SAS Institute Inc.). Disease-free survival was assessed with Kaplan-Meier curve and compared with log-rank test between categories of predictors (gender, age, antibody titers, adrenal function, associated diseases and number of diseases). Predictors found to be statistically significant at the 10\% level in the univariate Cox regression analysis were considered in a multivariate Cox regression model with backward stepwise selection of the predictors for survival free from diagnosis of AAD. The results were reported as $P$ value, hazard ratio (HR) and 95\% CI.

\section{Results}

Among 29 APS-1 patients, 20 were at Stage 0 and 9 were at Stage 1-2 during entry into the study. At the end of the follow-up, 24/29 (82.8\%) progressed to Stage 3-4 of AAD after a mean period of 3.9 years (range: 1-11 years) and required substitutive therapy. Furthermore, three other patients (10.3\%) progressed further from their initial stage but without developing Stages 3 or 4 of AAD (Fig. 1A).

In the case of patients with APS-2/APS-4, 88 of 114 were at Stage 0 while 26 were at Stage 1-2 during entry into the study. At the end of follow-up, 28/114 (24.6\%) progressed to Stage 3-4 after a mean period of 5.4 years (range: 1-19 years) and consequently required substitutive therapy. In addition, 11 other patients (9.6\%) advanced further from their initial stage, but none developed Stages 
Table 2 Stages of adrenal dysfunction in the natural history of AAD detectable by ACTH test in the ACA/21OHAb-positive patients.

\begin{tabular}{|c|c|c|c|}
\hline $\begin{array}{l}\text { Autoimmune Addison's } \\
\text { disease }\end{array}$ & Stage & $\begin{array}{l}\text { ACA and/or } \\
\text { 21-OHAbs }\end{array}$ & Symptoms \\
\hline Potential & 0 & + & Absent \\
\hline $\begin{array}{l}\text { Subclinical deficiency of } \\
\text { mineralocorticoids }\end{array}$ & 1 & + & Absent \\
\hline $\begin{array}{l}\text { Subclinical deficiency of } \\
\text { mineralocorticoids and } \\
\text { impaired reserve of } \\
\text { glucocorticoids }\end{array}$ & 2 & + & Absent \\
\hline $\begin{array}{l}\text { Subclinical deficiency of } \\
\text { mineralocorticoids and } \\
\text { of glucocorticoids }\end{array}$ & 3 & + & $\begin{array}{l}\text { Generally } \\
\text { absent or } \\
\text { non-specific }\end{array}$ \\
\hline $\begin{array}{l}\text { Clinical deficiency of } \\
\text { mineralocorticoids and } \\
\text { of glucocorticoids }\end{array}$ & 4 & + & Present \\
\hline
\end{tabular}

3 or 4 of AAD (Fig. 1B). All the patients at Stage 3 or Stage 4 were considered to have developed AAD and were on substitutive therapy as appropriate (Fig. 1).

The CI for AAD was $82.8 \%$ in APS-1 patients and $24.6 \%$ in patients with APS-2/APS-4 (23.5\% in those with APS-2 and $31.3 \%$ in those with APS-4). The CR of AAD in APS-1 patients was $94.2 \%$ and significantly increased compared to both, the CR of $68.1 \%$ in patients with APS-4 $(P<0.0001)$ and the CR of $35.2 \%$ in patients with APS-2 $(P<0.0001$; Fig. $2 \mathrm{~B}$ and Table 3$)$. The $\mathrm{CR}$ of $\mathrm{AAD}$ was comparable among APS-2 patients with AITD and DM-1 (42.9\%) to those with only AITD (31.7\%) or DM-1 (38.3\%) (Fig. 2E and Table 3).

With regard to the gender, for APS-1 patients, the CR for both males (100\%) and females (90.7\%) was very high, while for patients with APS-2/APS-4, the CR was significantly higher in males $(100 \%)$ than in females (31.1\%) $(P<0.0005)$ (Table 3).

\begin{tabular}{lll}
$\begin{array}{ll}\text { Plasma } \\
\text { renin }\end{array}$ & $\begin{array}{l}\text { Plasma } \\
\text { aldosterone }\end{array}$ \\
\cline { 1 - 1 } $\mathrm{N}$ & $\mathrm{N}$ \\
$\uparrow$ & $\mathrm{N} / \downarrow$ \\
& &
\end{tabular}

Plasma

ACTH

$\mathrm{N}$

N

N

\begin{tabular}{l}
$\begin{array}{l}\text { Plasma } \\
\text { cortisol }\end{array}$ \\
\hline $\mathrm{N}$ \\
$\mathrm{N}$
\end{tabular}

Plasma cortisol after i.v. ACTH $(250 \mu g)$

$\mathrm{N}$

$\mathrm{N}$

Impaired*

$\begin{array}{ccccc}\uparrow \uparrow & \downarrow & \uparrow & N / \downarrow & \text { No response } \\ \uparrow \uparrow \uparrow & \downarrow \downarrow & \uparrow \uparrow & \downarrow \downarrow & \text { No response }\end{array}$

Considering the age, the CR was high both in young (100\%) and in adult patients (73.3\%) with APS-1, while the CR was lower both in young (49.7\%) and adult patients (37.1\%) with APS-2/APS-4 (Table 3). With regard to adrenal autoantibody titers, the CR of AAD was similar among patients with high and those with low-medium titer (43.5\% and 59.4\%, respectively) (Fig. 2F and Table 3).

Analysis of AIRE gene showed that all the 24 ACA/21OHAb-positive patients with APS-1 who developed overt AAD had AIRE gene mutations, including one patient from control group who seroconverted during follow-up. Among ACA/21OHAb-positive patients with APS-1 who did not develop AAD, three of five had AIRE gene mutations. However, all the nine persistently ACA/21OHAb-negative patients with APS-1 who did not develop AAD also carried AIRE gene mutations.

In the control group, ACA/21OHAbs remained consistently negative during follow-up, except for one
A

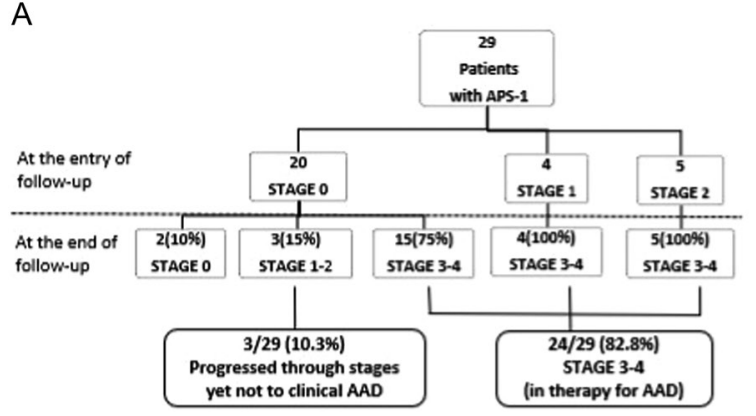

yet not to clinical $A A D$
B

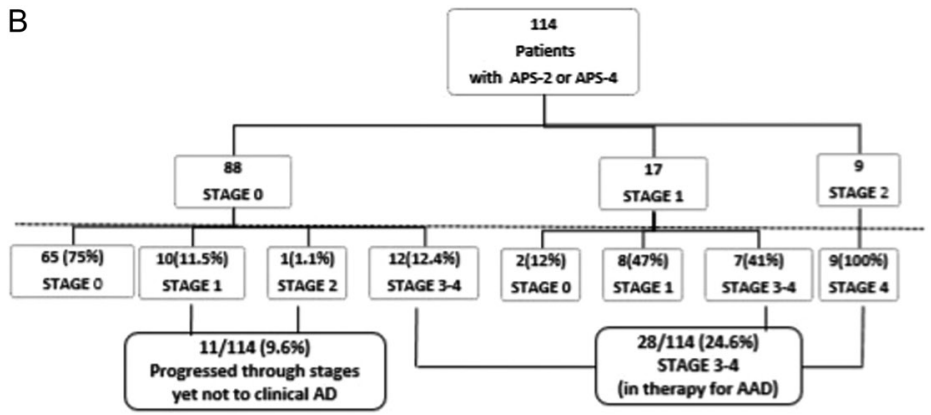

\section{Figure 1}

( $A$ and B) Assessment of adrenocortical function at entry into the study and at the end of follow-up in ACA/21OHAb-positive patients with APS-1 or APS-2/APS-4. 
female with APS-1 who developed AAD after 11 years of follow-up at 32 years of age. She was ACA negative when last tested 3 years before the diagnosis of AAD and was found to be ACA-positive at the diagnosis. In the group of ACA/21OHAb-positive patients, 140/143 (98\%) were persistently positive during the follow-up, while the remaining 3/143 (2.1\%) with APS-2 and initially having ACA titers 32, 64 and 128 (all at Stage 0 at entry) became seronegative after a median period of 4 years (range: $2-5$ years). These patients were followed in the group of negative controls and all three maintained normal adrenal function during this period (12-16 years).
In relation to the stages of adrenal cortex dysfunction, the CR of AAD in patients at Stage 0 was significantly higher in APS-1 (90.2\%) than in patients with APS-2/ APS-4 (29.5\%) $(P<0.0001$, HR: 9.1). At Stage 1, it was significantly higher in APS-1 patients $(100 \%)$ than in patients with APS-2/APS-4 $(46.9 \%) \quad(P<0.0001$, HR: 5.2). However, at Stage 2, it was $100 \%$ in both groups of patients (Fig. 2D and Table 3). In contrast to patients with APS-1, where all the cases at Stage 1 developed AAD (Fig. 1A), 8 of 17 patients (47\%) with APS-4/APS-4 at Stage 1 remained at the same stage for a long period (mean 192 months, range: $24-384$ months), 7 (41\%) progressed
A

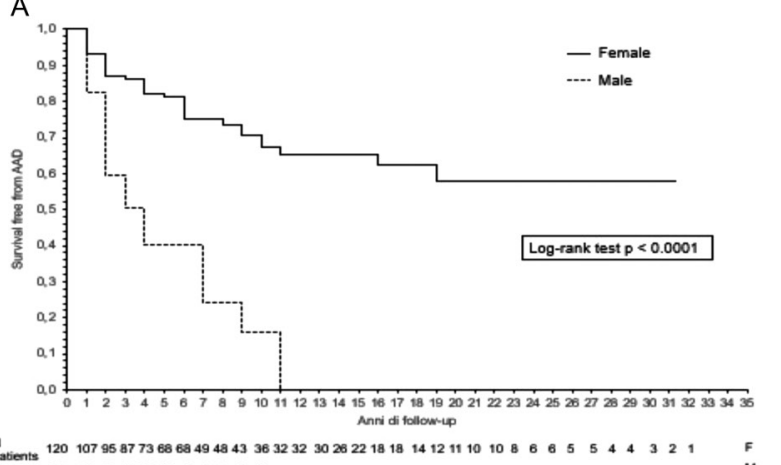

N
patents
at risk
23

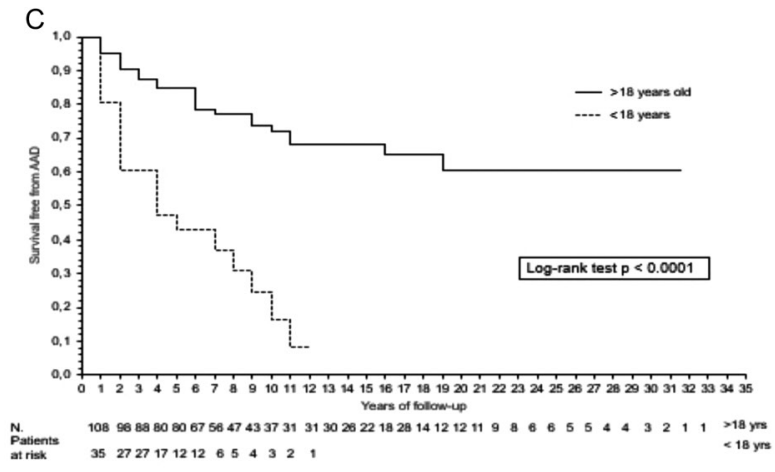

$\mathrm{E}$

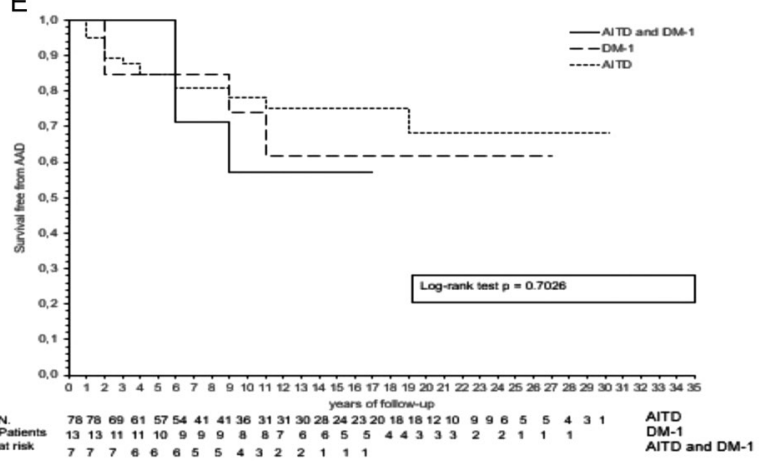

B

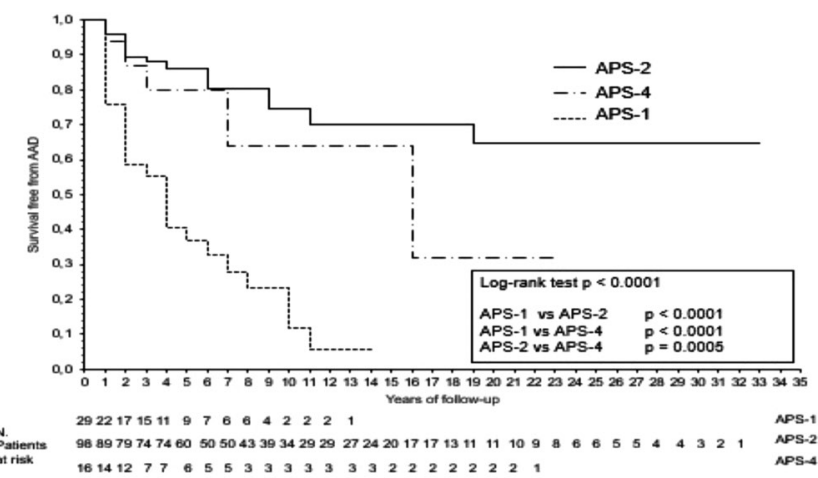

D

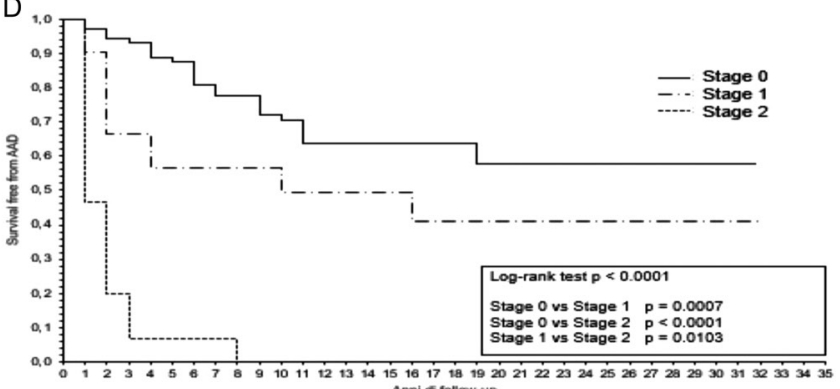

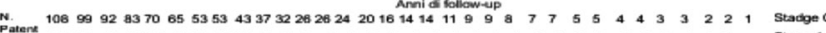

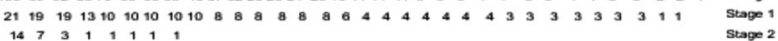

$\mathrm{F}$

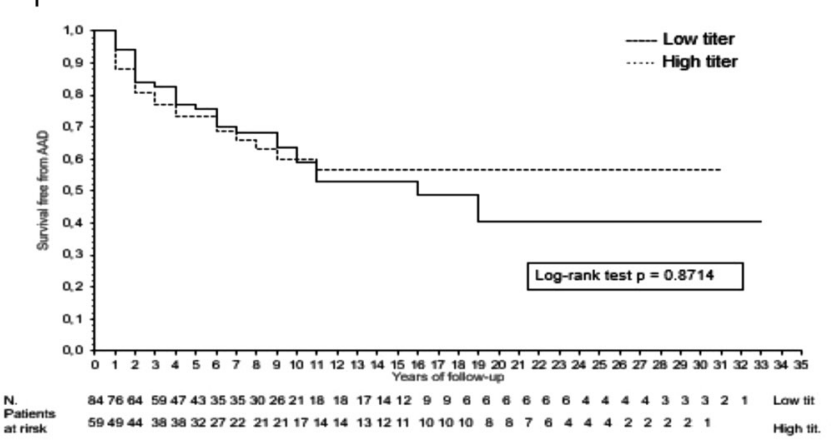

Figure 2

(A, B, C, D, E and F) Kaplan-Meier analysis of ACA/21OHAb-positive patients with the probability to survive free from overt AAD. 
Table 3 Cumulative incidence $(C I)$, cumulative risk $(C R)$ and annual incidence $(A I)$ of $A A D$ in different groups of $A C A / 21 O H A b$ positive patients with APS-1, APS-2 and APS-4.

\begin{tabular}{|c|c|c|c|c|c|}
\hline & Patients, $n$ & $\begin{array}{l}\text { Patients who developed } \\
\text { AAD, } n \\
\end{array}$ & CI $(\%)$ & CR $(\%)$ & Al $(\%)$ \\
\hline \multicolumn{6}{|l|}{ Pre-existing diseases } \\
\hline With APS-1 & 29 & 24 & 82.8 & 94.2 & 18.5 \\
\hline With APS-2/APS-4 & 114 & 28 & 24.6 & 38.7 & 11.2 \\
\hline With APS-2 & 98 & 23 & 23.5 & 35.2 & 2.5 \\
\hline AITD and DM-1 & 7 & 3 & 42.9 & 42.9 & 3.8 \\
\hline AITD alone & 78 & 16 & 20.5 & 31.7 & 2.3 \\
\hline DM-1 alone & 13 & 4 & 30.8 & 38.3 & 2.8 \\
\hline With APS-4 & 16 & 5 & 31.3 & 68.1 & 5.3 \\
\hline \multicolumn{6}{|l|}{ Gender } \\
\hline \multicolumn{6}{|l|}{ Males } \\
\hline With APS-1 & 10 & 10 & 100.0 & 100.0 & 38.5 \\
\hline With APS-2/APS-4 & 13 & 8 & 61.5 & 100.0 & 11.7 \\
\hline \multicolumn{6}{|l|}{ Females } \\
\hline With APS-1 & 19 & 14 & 73.8 & 90.7 & 13.3 \\
\hline With APS2/APS-4 & 101 & 20 & 19.8 & 31.1 & 2.1 \\
\hline \multicolumn{6}{|l|}{ Age } \\
\hline \multicolumn{6}{|l|}{ Young } \\
\hline With APS-1 & 20 & 19 & 95.0 & 100.0 & 25.3 \\
\hline With APS-2/APS-4 & 13 & 4 & 30.8 & 49.7 & 6.3 \\
\hline Adult & 110 & 29 & 26.4 & 40.0 & 2.9 \\
\hline With APS-1 & 9 & 5 & 55.6 & 73.3 & 9.1 \\
\hline With APS-2/APS-4 & 101 & 24 & 23.8 & 37.1 & 2.5 \\
\hline \multicolumn{6}{|l|}{ Adrenal function } \\
\hline \multicolumn{6}{|l|}{ Stage 0} \\
\hline With APS-1 & 20 & 15 & 75.0 & 90.2 & 14.7 \\
\hline With APS-2/APS-4 & 88 & 12 & 13.6 & 29.5 & 1.4 \\
\hline \multicolumn{6}{|l|}{ Stage 1} \\
\hline With APS-1 & 4 & 4 & 100.0 & 100.0 & 26.7 \\
\hline With APS-2/APS-4 & 17 & 7 & 41.2 & 46.9 & 4.1 \\
\hline \multicolumn{6}{|l|}{ Stage 2} \\
\hline With APS-1 & 5 & 5 & 100.0 & 100.0 & 38.5 \\
\hline With APS-2/APS-4 & 9 & 9 & 100.0 & 100.0 & 56.3 \\
\hline \multicolumn{6}{|l|}{ Adrenal antibody titer } \\
\hline Low-medium & 84 & 31 & 36.9 & 59.4 & 4.7 \\
\hline High & 59 & 21 & 35.6 & 43.5 & 4.3 \\
\hline
\end{tabular}

Al, annual incidence; AITD, autoimmune thyroid diseases; APS, autoimmune polyendocrine syndromes; $\mathrm{Cl}$, cumulative incidence; CR, cumulative risk; DM-1, type 1 diabetes mellitus.

to Stages 3-4 and 2 (12\%) improved to Stage 0 (Fig. 1B). These observations suggest that the point of no return is Stage 1 in patients with APS-1, while it is Stage 2 in patients with APS-2/APS-4.

Progression of the disease varied in different patients, some patients starting at Stage 0 or Stage 1 advanced sequentially through all stages. In the case of other patients, this pattern was not observed; for example, some started at Stage 0 and were next observed at Stage 2 and Stage 4, but not at Stage 1 and 3. Furthermore, elevated ACTH levels with normal renin and aldosterone levels were not observed in any of our patients.

Disease-free survival from AAD in patients with APS-1, APS-2 and APS-4, groups of patients with ACA and/or 210HAbs were estimated using Kaplan-Meier curves and summarized in Fig. 2A, B, C, D, E and F. With regard to the length of time for development of $\mathrm{AAD}$, the vast majority of the patients $(50 / 52,96.2 \%)$ progressed to AAD within 11 years from the first detection of ACA/21OHAbs. Only 2 of 52 (3.8\%) developed AAD after 11 years of follow-up (one after 17 and the other one after 19 years). Moreover, the progression to AAD was faster in patients with APS-1 (mean period: 3.9 years; range: 1-11) compared with patients with APS-2/APS-4 (mean period 5.4 years, range 1-19). None of the 11 patients with APS-2/APS-4 who were followed for longer than 19 years developed AAD.

We carried out univariate and multivariate analyses of the risk for the development of AAD according to age, gender, adrenal function and adrenal antibody 
titer, in patients with ACA/21OHAbs at entry to the end of follow-up using Cox proportional hazards model.

Based on univariate analysis, four parameters were significantly associated with the future development of AAD: (a) young age, (b) male gender, (c) impaired adrenal function and (d) coexistence of APS-1. In contrast, co-occurrence with AITD and/or DM-1 (i.e., APS-2) and titer of adrenal autoantibody were not associated with the progression to AAD (Fig. 2A, B, C, D, E and F; Table 4).

However, based on multivariate analysis, only three of these parameters, that is, gender, type of pre-existing autoimmune diseases and adrenal cortical function, were found to be independent risk factors (Table 4). In multivariate analysis, adjusted HR by Cox model for the development of AAD were 3.6 for males (CI: 1.9-6.9), 4.3 for APS-1 (CI: 1.6-11.5), 8.5 for Stage 2 (CI: 4.1-17.7) and 2.7 for Stage 1 (CI: 1.3-5.6)), while age was not relevant for the progression to AAD. These data were used to plot a graphic model (Fig. 3A, B, C, D, E and F) which could be used to estimate the probability of survival free from AAD in patient with adrenal autoantibodies. For example, in the case of a female with APS-2 with initial adrenal Stage 0,1 or 2 , the probability of survival free of AAD after 5 years can be read off the vertical axis on the graph (and is equal to 93, 81 and 52\%, respectively) (Fig. 3C). In contrast, a male with APS- 2 and initial Stage 0, 1 or 2 will have a probability of surviving free from AAD after 5 years of 76, 46 and 9\%, respectively (Fig. 3D).

\section{Discussion}

In this study, we report the outcome of the follow-up of $143 \mathrm{ACA} / 21 \mathrm{OHAb}$-positive patients without clinical AAD. To date, this represents the greatest cohort with the longest follow-up period (median 10 years, with a group of 12 patients followed for over 19 years and a maximum period of observation of 33 years). We have also reassessed the previously reported prognostic value of various clinical and biochemical factors for disease progression (26). Furthermore, we analyzed the prognostic values separately for ACA/21OHAb-positive patients with APS-1, APS-2 and APS-4.

ACA/21OHAb-positive patients with APS-1 demonstrated the highest risk and the more accelerated progression toward clinical AAD, independently from gender and stage of adrenal dysfunction at entry into the follow-up when compared to the group of ACA/21OHAbpositive patients with APS- 2 or APS-4. Furthermore, the $\mathrm{CR}$ and $\mathrm{AI}$ of clinical AAD were very high for both males and females with APS-1, while in patients with APS- 2 or APS-4, the CR and AI of AAD was significantly higher in males than in females. This suggests that the presence of AIRE gene mutations in APS-1 is an important risk factor in promoting clinical AAD development, independently from the gender. On the contrary, male gender becomes an important predisposing feature in patients with HLArelated APS-2/APS-4. The multivariate analysis confirmed that the male gender is an independent risk factor for AAD for all APS-2/APS-4 patients (Figs 2A and 3B, D, F).

Table 4 Univariate and multivariate analysis using Cox proportional hazards model for the development of overt AAD according to different variables (age, gender, adrenal function at entry, pre-existing autoimmune disease, adrenal antibody titers).

\begin{tabular}{|c|c|c|c|c|}
\hline Variable & Univariate analysis, HR $(95 \% \mathrm{Cl})$ & $\boldsymbol{P}$ & Multivariate analysis, HR $(95 \% \mathrm{Cl})$ & $\mathbf{P}$ \\
\hline Age (years) & & $<0.0001$ & & \\
\hline$\leq 18$ & $4.445(2.506-7.884)$ & & - & \\
\hline$>18$ & 1 & & & \\
\hline Gender & & $<0.0001$ & & $<0.0001$ \\
\hline Male & $4.403(2.444-7.934)$ & & $3.628(1.903-6.915)$ & \\
\hline Female & 1 & & 1 & \\
\hline Adrenal dysfunction & & $<0.0001$ & & $<0.0001$ \\
\hline Stage 2 & $13.326(6.492-27.354)$ & & $8.475(4.056-17.706)$ & \\
\hline Stage 1 & $2.260(1.118-4.568)$ & & $2.723(1.325-5.594)$ & \\
\hline Stage 0 & 1 & & 1 & \\
\hline Pre-existing diseases & & $<0.0001$ & & 0.0039 \\
\hline With APS-1 & 1 & & $4.288(1.597-11.508)$ & \\
\hline With APS-2 & $0.171(0.095-0.310)$ & & $1.122(0.397-3.169)$ & \\
\hline With APS-4 & $0.321(0.122-0.846)$ & & 1 & \\
\hline Adrenal antibody titer & & 0.8756 & & \\
\hline High & $0.957(0.549-1.666)$ & & & \\
\hline Low-medium & 1 & & & \\
\hline
\end{tabular}


A

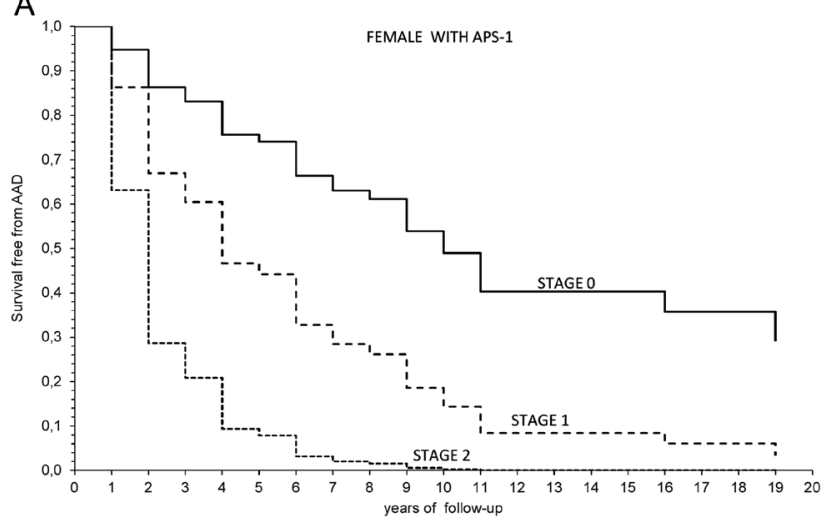

C

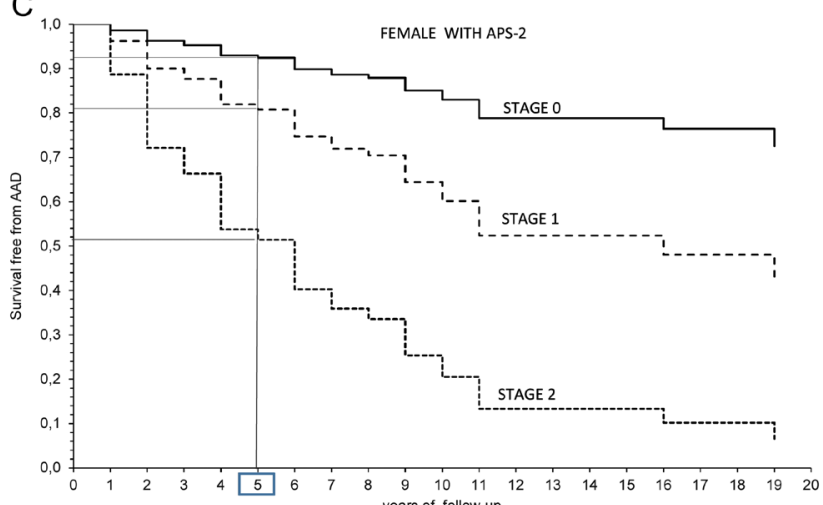

E

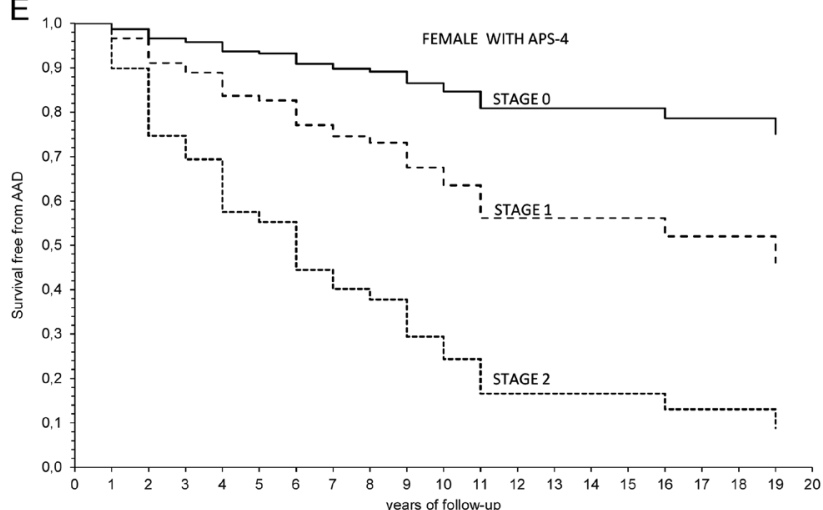

B

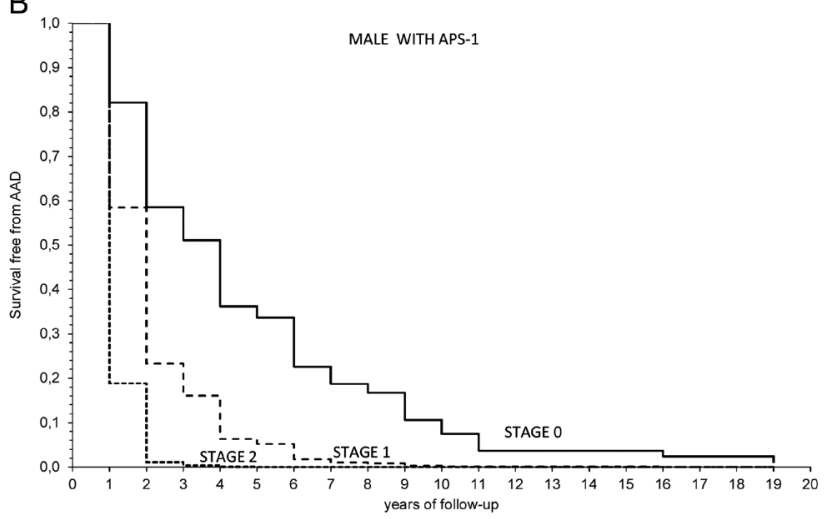

$\mathrm{D}_{1,0}$

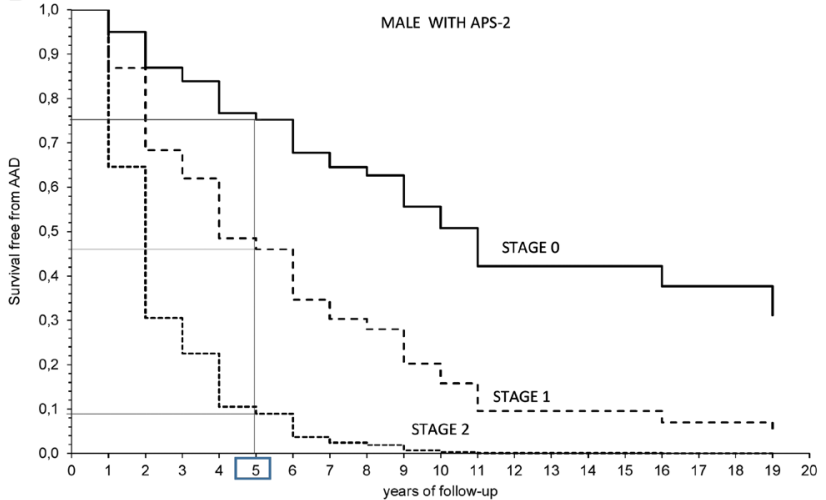

$\mathrm{F}$

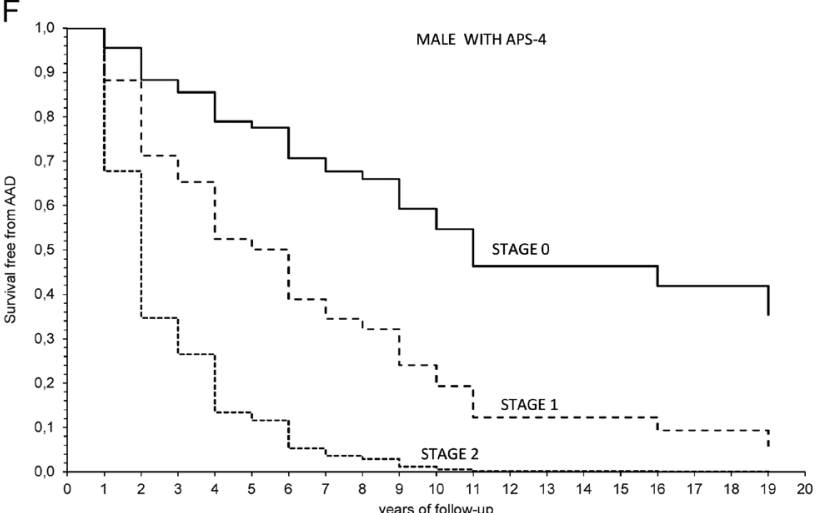

\section{Figure 3}

(A, B, C, D, E and F) Graphic model for estimating the probability to survive free of AAD for ACA/21OHAb-positive patients on the basis of the three independent risk factors: gender (left column for females and right column for males), coexistence of autoimmune diseases (APS-1, APS-2, APS-4) and stages of adrenal dysfunction $(0,1,2)$ at the start of follow-up.

Overall, ACA/21OHAb-positive patients with young age at entry showed high CR of AAD. However, a great number of young patients are affected by APS-1. When CR was analyzed separately for young patients without APS-1, CR was low and similar to that of adult patients without APS-1. The multivariate analysis confirmed that the age is not an independent risk factor of AAD for all the APS patients.
In our previous studies, we suggested that Stage 1 of adrenal dysfunction was the point of no return for progression to $\operatorname{AAD}(14,15,28)$. However, sporadically ACA-positive patients at Stage 1-2 may improve to normal adrenal function, either spontaneously or after high doses of steroids (30). One reported patient remained in remission for more than 100 months after steroid treatment (31). In the present study, we have 
confirmed that the Stage 2 of adrenal cortical dysfunction at the beginning of the follow-up is an important and independent risk factor for the progression to AAD for all the patients with APS-1 or APS-2/APS-4 (Fig. 2E). However, we have also demonstrated that the progression to AAD differs between patients with APS-1 and those with APS-2/ APS-4. While all APS- 1 patients at Stage 1 developed clinical AAD, less than one-half of APS-2/APS-4 patients at Stage 1 progressed to clinical AAD, one-half remained at Stage 1 for a long time, and some patients even regained normal adrenal function improving to Stage 0 (Fig. 1C). These observations suggest that the point of no return to adrenal failure in APS-1 patients may be as early as Stage 1, while in patients with APS-2/APS-4, this point is at Stage 2. This would justify a closer monitoring of ACA/21OHAbpositive patients with APS- 1 at Stage 0 or 1 . In contrast, ACA/21OHAb-positive patients with APS-2/APS-4 at Stage 0 or 1 may be followed up less frequently, since their risk of progression to AAD is much lower and sometimes their adrenal dysfunction may also improve.

In our study, the progression from the initial to the following stages of adrenal impairment varied greatly among the patients. This could be related to the rate of progression and the interval of the biochemical testing. However, none of our patients presented with increased baseline ACTH and normal renin and aldosterone values, as described by others (20). It should be noted that such combination of biochemical findings is not typical in patients with adrenal autoimmune dysfunction and has been only sporadically reported $(20,32)$.

We have observed that during follow-up, some $\mathrm{ACA} / 21 \mathrm{OHAb}$-positive patients became negative, while the one negative control seroconverted to positive. Therefore, it would seem as a good practice to periodically detect the autoantibodies in the patients at risk of AAD.

This study included three groups of ACA/21OHAbpositive patients with different combinations of associated autoimmune diseases: 29 with APS-1, 98 with APS- 2 and 16 with APS-4. We demonstrated that the risk of developing AAD is higher in patients with APS-1, intermediate in those with APS- 4 and lower in those with APS- 2 .

Furthermore, for the first time, we had the opportunity to define the separate risk in patients with different combination of associated autoimmune diseases in the context of APS-2, such as in patients with AITD or DM-1 or both, and we demonstrated that these patients had a similar low risk of developing AAD irrespective of the co-occurring diseases (Fig. 2E).

During follow-up, the great majority of patients progressed to AAD within 11 years from the time of their first adrenal autoantibody detection and only a minority developed AAD between 11 and 19 years of follow-up. The progression was faster in patients with APS-1 (mean 3.9 years) and all of them developed AAD within 11 years of follow-up. On the contrary, in patients with APS2/APS4 , the progression was slower (mean of 5.4 years) and AAD developed within 19 years. None of the 11 patients with APS-2 or APS-4 developed AAD after 19 years (Fig. 2B). Therefore, the risk of developing AAD is very low after 11 years of follow-up and is virtually absent after 19 years. Consequently, patients who have not yet developed AAD after 19 years of follow-up can be advised that they are no longer at risk of progression to the disease and that further laboratory investigations are not necessary.

In the majority of APS-1 patients, the condition presents in young individuals with $\mathrm{CC}$ and/or $\mathrm{CH}$ (2). However, in some patients, the first presentation can be more heterogeneous with other clinical manifestations such as enamel hypoplasia, enteropathy, POI, periodic fever, non-infectious keratitis or autoimmune hepatitis $(7,8,9)$. For these reasons, patients with clinical manifestations suggestive of APS- 1 should be screened initially for interferon autoantibodies and, if positive, followed by analyses for AIRE gene mutations to confirm or not the diagnosis of APS-1 (7). If AAD is not present at the diagnosis of APS-1, it would be most appropriate to test these patients for ACA/21OHAbs, which are detectable in up to $47 \%$ of the cases $(2,14)$, and the positive patients are at very high risk of progression to AAD.

To date, there have been conflicting reports on the relationship between Class II HLA and the risk of progression to AAD in patients with 21OHAbs $(15,17,19$, 26). In our previous study, an association between Class II HLA haplotypes and progression to AAD was not found (26), and therefore, this evaluation has not been included in the present investigation. One study reported that MHC Class I chain-related (MIC-A) polymorphism was increased in patients with overt $\mathrm{AAD}$, although only in the presence of HLA-DR3/DQ2 (33). In addition, in a study of a selected population of patients with DM-1 who were positive for 21OHAbs, 17 of 47 were homozygous for MIC-A5.1 allele (19). Six out of these 17 patients developed overt AAD indicating that MIC-A5.1 haplotype may increase the risk of progression to clinical AAD in patients with DM-1. In our study, there were a low number of patients who were DM-1 positive for 21OHAbs, and therefore, MIC-A testing has not been carried out. Nonetheless, our study demonstrates that the presence of AIRE gene mutations may be an important factor conferring the risk of $\mathrm{AAD}$ in ACA/21OHAb-positive individuals. 
In ACA/21OHAb-positive patients, we identified only three independent risk factors for developing clinical AAD (male gender, APS-1, and impaired adrenal function at patient enrollment) using the multivariate analysis (Table 4). The Kaplan-Meier curves in patients with different titers of ACA did not reveal differences in the risk of progression to $\mathrm{AAD}$, in contrast to our previous observations (26). This indicates that ACA/21OHAbs are serological rather than pathogenic markers of adrenal failure and that the disease is primarily cell mediated.

Based on the three multivariate factors inducing AAD, a graphic model for estimating the probability of survival free of AAD has been proposed for males and females with APS-1, APS- 2 or APS-4, and Stages 0St, 1 or 2 of adrenal dysfunction at the beginning of follow-up (Fig. 3).

The model should be helpful for endocrine practitioners to aid in the regular assessment of ACA/21OHAb-positive patients and timely initiation of substitutive therapy with the aim to prevent adrenal crisis in patients with ongoing AAD. It could be helpful in assessing the risk of developing AAD in patients enrolled for clinical trials for treatments aimed to modify the natural history of AAD. Improved awareness of the risk of progression to AAD should have an impact on the quality of care of patients and help in developing better strategies and new drugs for preserving adrenal function and delaying the onset of adrenal failure.

In conclusion, we found that the $\mathrm{CR}$ of developing AAD is much higher in ACA/21OHAb-positive patients with APS- 1 than in those with APS-4 or APS-2.

\section{Declaration of interest}

J F, S C and B R S are employees of RSR Ltd. RSR Ltd is a developer of medical diagnostics, including kits for measuring 21-hydroxylase autoantibodies. The other authors have no conflict of interest.

\section{Funding}

This study was supported in part by a grant from the EU Seventh Framework Programme, Euradrenal project: Pathophysiology and Natural Course of Autoimmune Adrenal Failure in Europe: Grant No. 2008-201167, and by a grant ex $60 \%$ from the University of Padua.

\section{References}

1 Oelkers W. Adrenal insufficiency. New England Journal of Medicine 1996335 1206-1212. (https://doi.org/10.1056/ NEJM199610173351607)

2 Betterle C, Dal Pra C, Mantero F \& Zanchetta R. Autoimmune adrenal insufficiency and autoimmune polyendocrine syndromes: autoantibodies, autoantigens, and their applicability in diagnosis and disease prediction. Endocrine Review 200223 327-364. (https://doi. org/10.1210/edrv.23.3.0466)
3 Charmandari E, Nicolaides NC \& Chrousos GP. Adrenal insufficiency. Lancet 2014383 2152-2167. (https://doi.org/10.1016/S01406736(13)61684-0)

4 Olafsson AS \& Sigurjonsdottir HA. Increasing prevalence of Addison's disease. Results from a Nationwide study. Endocrine Practice 201622 30-35. (https://doi.org/10.4158/EP15754.OR)

5 Betterle C, Scarpa R, Garelli S, Morlin L, Lazzarotto F, Presotto F, Coco G, Masiero S, Parolo A, Albergoni MP et al. Addison's disease: a survey on 633 patients in Padova. European Journal of Endocrinology 2013169 773-784. (https://doi.org/10.1530/EJE-13-0528)

6 Eisenbarth GS \& Gottlieb PA. Autoimmune polyendocrine syndromes. New England Journal Medicine $20043502068-2079$. (https://doi.org/10.1056/NEJMra030158)

7 Husebye ES, Anderson MS \& Kampe O. Autoimmune polyendocrine syndrome. New England Journal of Medicine 2018378 1132-1141. (https://doi.org/10.1056/NEJMra1713301)

8 Perheentupa J. Autoimmune polyendocrinopathy-candidiasisectodermal dystrophy. Journal of Clinical Endocrinology and Metabolism $2006912843-2845$.

9 Orlova EM, Sozaeva LS, Kareva MA, Oftedal BE, Wolff ASB, Breivik L, Zakharova EY, Ivanova ON, Kämpe O, Dedov II et al. Expanding the phenotypic and genotypic landscape of autoimmune polyendocrine syndrome type 1. Journal of Clinical Endocrinology and Metabolism 2017102 3546-3556. (https://doi.org/10.1210/jc.2017-00139)

10 Blizzard RM, Kyle MA, Chandler RW \& Hung W. Adrenal antibodies in Addison's disease. Lancet 19622 901-903. (https://doi. org/10.1016/S0140-6736(62)90681-5)

11 Betterle C, Coco G \& Zanchetta R. Adrenal cortex autoantibodies in subjects with normal adrenal function. Best Practice and Research Clinical Endocrinology and Metabolism 200519 85-89. (https://doi. org/10.1016/j.beem.2004.11.008)

12 Winqvist O, Karlsson FA \& Kampe O. 21-Hydroxylase, a major autoantigen in idiopathic Addison's disease. Lancet 1992339 1559-1562. (https://doi.org/10.1016/0140-6736(92)91829-W)

13 Baumann Antczak A, Wedlock N, Bednarek J, Kiso Y, Krishnan H, Fowler S, Rees Smith B \& Furmaniak J. Autoimmune Addison's disease and 21-hydroxylase. Lancet 1992340 429-430. (https://doi. org/10.1016/0140-6736(92)91513-8)

14 Betterle C, Volpato M, Rees Smith B, Furmaniak J, Chen S, Zanchetta R, Greggio NA, Pedini B, Boscaro M \& Presotto F. II. Adrenal cortex and steroid 21-hydroxylase autoantibodies in children with organ-specific autoimmune diseases: markers of high progression to clinical Addison's disease. Journal of Clinical Endocrinology and Metabolism 199782 939-942. (https://doi. org/10.1210/jcem.82.3.3849)

15 Betterle C, Volpato M, Rees Smith B, Furmaniak J, Chen S, Greggio NA, Sanzari M, Tedesco F, Pedini B, Boscaro M et al. I. Adrenal cortex and steroid 21-hydroxylase autoantibodies in adult patients with organ specific autoimmune diseases: markers of low progression to clinical Addison's disease. Journal of Clinical Endocrinology and Metabolism 199782 932-938. (https://doi. org/10.1210/jcem.82.3.3819)

16 Falorni A, Laureti S, Nikoshkov A, Picchio ML, Hallengren B, Vandewalle CL, Gorus FK, Tortoioli C, Luthman H, Brunetti P et al. 21-Hydroxylase autoantibodies in adult patients with endocrine autoimmune diseases are highly specific for Addison's disease. Belgian Diabetes Registry. Clinical and Experimental Immunology 1997107 341-346. (https://doi.org/10.1111/j.1365-2249.1997.262ce1153.x)

17 Yu L, Brewer KW, Gates S, Wu A, Wang T, Babu SR, Gottlieb PA, Freed BM, Noble J, Erlich HA et al. DRB1*04 and DQ alleles: expression of 21-hydroxylase autoantibodies and risk of progression to Addison's disease. Journal of Clinical Endocrinology and Metabolism 199984 328-335. (https://doi.org/10.1210/jcem.84.1.5414)

18 Peterson P, Salmi H, Hyöty H, Miettinen A, Ilonen J, Reijonen H, Knip M, Akerblom HK \& Krohn K. Steroid 21-hydroxylase 
autoantibodies in insulin-dependent diabetes mellitus. Childhood Diabetes in Finland (DiMe) Study Group. Clinical Immunology and Immunopathology 199782 37-42. (https://doi.org/10.1006/ clin.1996.4277)

19 Barker JM, Ide A, Hostetler C, Yu L, Miao D, Fain PR, Eisenbarth GS \& Gottlieb PA. Endocrine and immunogenetic testing in individuals with type 1 diabetes and 21-hydroxylase autoantibodies: Addison's disease in a high-risk population. Journal of Clinical Endocrinology and Metabolism 200590 128-134. (https://doi.org/10.1210/jc.2004-0874)

20 Baker PR, Nanduri P, Gottlieb PA, Yu L, Klingensmith GJ, Eisenbarth GS \& Barker JM. Predicting the onset of Addison's disease: ACTH, renin, cortisol and 21-hydroxylase autoantibodies. Clinical Endocrinology 201276 617-624. (https://doi.org/10.1111/j.13652265.2011.04276.x)

21 Del Pilar Larosa M, Chen S, Steinmaus N, Macrae H, Guo L, Masiero S, Garelli S, Dalla Costa M, Bossowski A, Furmaniak J et al. A new ELISA for autoantibodies to steroid 21-hydroxylase. Clinical Chemistry and Laboratory Medicine 201856 933-938. (https://doi. org/10.1515/cclm-2017-0456)

22 Betterle C, Zanette F, Zanchetta R, Pedini B, Trevisan A, Mantero F \& Rigon F. Complement-fixing adrenal autoantibodies as a marker for predicting onset of idiopathic Addison's disease. Lancet 19831 1238-1241. (https://doi.org/10.1016/S0140-6736(83)92695-8)

23 Ahonen P, Miettinen A \& Perheentupa J. Adrenal and steroidal cell antibodies in patients with autoimmune polyglandular disease type I and risk of adrenocortical and ovarian failure. Journal of Clinical Endocrinology and Metabolism 198764 494-500. (https://doi. org/10.1210/jcem-64-3-494)

24 Betterle C, Scalici C, Presotto F, Pedini B, Moro L, Rigon F \& Mantero F. The natural history of adrenal function in autoimmune patients with adrenal autoantibodies. Journal of Endocrinology 1988 117 467-475. (https://doi.org/10.1677/joe.0.1170467)

25 Laureti S, De Bellis AM, Muccitelli VI, Calcinaro F, Bizzaro A, Rossi R, Bellastella A, Santeusanio F \& Falorni A. Levels of adrenocortical autoantibodies correlate with the degree of adrenal dysfunction in subjects with preclinical Addison's disease. Journal of Clinical Endocrinology and Metabolism 199883 3507-3511. (https://doi. org/10.1210/jcem.83.10.5149)

26 Coco G, Dal Pra C, Presotto F, Albergoni MP, Canova C, Pedini B, Zanchetta R, Chen S, Furmaniak J, Rees Smith B et al. Estimated risk for developing autoimmune Addison's disease in patients with adrenal cortex autoantibodies. Journal of Clinical Endocrinology and Metabolism 200691 1637-1645. (https://doi.org/10.1210/jc.20050860)

27 Eason RJ, Croxon MS, Perry MC \& Somerfield SD. Addison's disease, adrenal autoantibodies and computerized adrenal tomography. New Zealand Medical Journal 198295 569-573.

28 Betterle C, Garelli S, Presotto F \& Furmaniak J. From appearance of adrenal autoantibodies to clinical symptoms of Addison's disease: natural history. Frontiers of Hormonal Research 201646 133-145. (https://doi.org/10.1159/000443872)

29 Cervato S, Mariniello B, Lazzarotto F, Morlin L, Zanchetta R, Radetti G, De Luca F, Valenzise M, Giordano R, Rizzo D et al. Evaluation of these autoimmune regulator (AIRE) gene mutations in a cohort of Italian patients with autoimmune-polyendocrinopathycandidiasis-ectodermal-dystrophy (APECED) and in their relatives. Clinical Endocrinology 200970 421-428. (https://doi.org/10.1111/ j.1365-2265.2008.03318.x)

30 De Bellis A, Bizzarro A, Rossi R, Paglionico VA, Criscuolo T, Lombardi G \& Bellastella A. Remission of subclinical adrenocortical failure in subjects with adrenal autoantibodies. Journal of Clinical Endocrinology and Metabolism 199376 1002-1007. (https://doi. org/10.1210/jcem.76.4.8473373)

31 De Bellis AM, Falorni A, Laureti S, Perrino S, Coronella C, Forini F, Bizzarro E, Bizzarro A, Abbate G \& Bellastella A. Time course of 21-hydroxylase antibodies and long-term remission of subclinical autoimmune adrenalitis after corticosteroid therapy: case report. Journal of Clinical Endocrinology and Metabolism 200186 675-678. (https://doi.org/10.1210/jcem.86.2.7212)

32 Manso J, Pezzani R, Scarpa R, Gallo N \& Betterle C. The natural history of autoimmune Addison's disease with a non-classical presentation: a case report and review of literature. Clinical Chemistry and Laboratory Medicine 201856 896-900. (https://doi.org/10.1515/ cclm-2017-1108)

33 Gambelunghe G, Falorni A, Ghaderi M, Laureti S, Tortoioli C, Santeusanio F, Brunetti P \& Sanjeevi CB. Microsatellite polymorphism of the MHC class I chain-related (MIC-A and MIC-B) genes marks the risk for autoimmune Addison's disease. Journal of Clinical Endocrinology and Metabolism 199984 3701-3707. (https:// doi.org/10.1210/jcem.84.10.6069)

Received 13 April 2018

Revised version received 2 December 2018

Accepted 2 January 2019 